\title{
Santé des enfants en Mauritanie
}

\section{Jean Martin}

Dr méd., membre de la rédaction

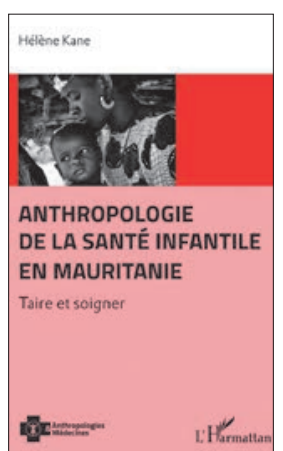

Hélène Kane

Anthropologie de la santé infantile

en Mauritanie - Taire et soigner

Paris: L’Harmattan; 2018.

274 pages.

ISBN 978-2-343-15964-5

Cet ouvrage introduit profondément dans la vie des familles mauritaniennes. Principalement dans la capitale Nouakchott, où l'auteure a étudié les croyances et les pratiques en rapport avec la santé et la maladie des enfants. Il importe de savoir que «la pluralité culturelle des enfances tient à la diversité de la société, composée de Maures et de populations dites négro-mauritaniennes - pulaar» (d’origine notamment sénégalaise). Même si c'est le milieu pulaar qui a été étudié de manière approfondie, la comparaison des deux ensembles est souvent faite dans les recherches présentées.

«Les enfants sont diversement confrontés à des problèmes d'accès aux richesses, à la scolarisation et aux soins.» "Autour des trajectoires de maladie de quelques enfants, nous avons exploré les configurations familiales, les représentations des maladies et les logiques interprétatives.» Hélène Kane documente remarquablement parcours, croyances, différences et inégalités, au travers de vignettes de la vie - parfois de la mort d'enfants et, notamment, de leurs mères. Confrontations aux multiples dimensions de la maladie, aux problèmes de relation avec les professionnels - le dialogue n'étant trop souvent ni empathique ni à égalité. «Les jeunes mères sont aux prises avec des faisceaux de normes exigeantes et parfois contradictoires.» S'ajoutent les complexités administratives et difficultés d'accès - qui pourraient être levées par des apports financiers additionnels, dont un grand nombre de familles ne disposent pas. On se souvient que des formes d'esclavage persistent en Mauritanie (malgré une quatrième abolition officielle, en 1980).

Travail d'une anthropologue, considérant la maladie comme un "fait social total», l'ouvrage va «au-delà de la mise à disposition de services géographiquement et économiquement accessibles. L'accessibilité sociale et cognitive est questionnée» (on me permettra - J.M. de relever que, en principe, la santé publique devrait elle aussi avoir une telle vision holistique). Cinq chapitres. Le premier sur l'anthropologie de l'enfance dans le pays. Le suivant sur les problématiques liées à santé et maladie en milieu haal pulaar (à savoir négro-mauritanien); y sont décrites plusieurs classes de maladie et les croyances et pratiques à leur endroit. Le troisième a pour titre «Les guérisseurs dans l'ombre et au grand jour» et le quatrième décrit le système de santé à disposition (avec «les articulations et décalages d'une étape de soins à l'autre»). Le dernier s'attache aux trajectoires et expériences en milieu hospitalier, avec des observations qui peuvent laisser songeur.

Quelques éclairages: «Les systèmes de santé comportent parfois des violences: absence d'attention pour le soulagement de la douleur ou négligence.» Il y a "dépendance de la qualité des soins du statut social, affectif et décisionnel donné à l'enfant». Ce qui n'étonnera en rien, étant une constante - à des degrés variables bien sûr - dans toutes les sociétés, même riches et relativement égalitaires. «La méfiance, l'impression que les coûts sont trop élevés, la critique et la comparaison avec les normes étrangères dominent les perceptions des Mauritaniens.» Intéressant: «Les enfants que nous avons rencontrés interviennent sur leur prise en charge par l'intermédiaire de sollicitations habiles et discrètes. Ils apprennent à moduler leurs plaintes.» En rapporte avec le sous-titre de l'ouvrage: «La prudence face au pouvoir des mots traverse l'ensemble de la société mauritanienne.»

Facette ethnologique interpelante: «Les femmes pulaar participent à l'économie domestique par divers apports. Les femmes maures elles ne sont pas tenues de contribuer à l'entretien de leur famille; les maris doivent assurer toutes les dépenses et tendent à présenter leurs femmes comme oisives et inconsistantes. Cela a pour corollaire une certaine déresponsabilisation des femmes. Un travail chez les Tamasheq (Touareg) du Mali constate une négligence comparable des femmes nobles, dans une société où elles accroissent leur prestige en travaillant le moins possible, y compris soins aux enfants.»

Anthropologie de la santé infantile en Mauritanie intéressera les professionnels de la santé et du social, aussi chez nous bien sûr dans la mesure où les patients provenant d'autres pays et cultures sont nombreux. 